\title{
Polyakov conjecture and $2+1$ dimensional gravity coupled to particles
}

\section{Luigi Cantini}

Scuola Normale Superiore,Pisa and INFN Sezione di Pisa, Italy

E-mail: 'cantini@df unipi.it'

\section{Pietro Menotti* ${ }^{\dagger}$}

Dipartimento di Fisica della Università, Pisa and INFN Sezione di Pisa, Italy E-mail: menotti@df unipi.it'

\section{Domenico Seminara}

Dipartimento di Fisica della Università, Firenze and INFN Sezione di Firenze, Italy E-Mail: 'seminara@fi.infn.it'

Abstract: A proof is given of Polyakov conjecture about the auxiliary parameters of the $S U(1,1)$ Riemann-Hilbert problem for general elliptic singularities. Such a result is related to the uniformization of the the sphere punctured by $n$ conical defects. Its relevance to the hamiltonian structure of $2+1$ dimensional gravity in the maximally slicing gauge is stressed.

1. We shall deal with the proof of a conjecture put forward by Polyakov [I, ] about the accessory parameters of the Riemann- Hilbert problem and with the role such a conjecture plays in $2+1$ dimensional gravity coupled to particles on an open universe. Such a theory when formulated in the maximally slicing gauge (Dirac gauge) [2] shows a deep connection with the Riemann- Hilbert problem which consists in determining a fuchsian differential equation whose independent solutions transform according to a given representation of the $S L(2 C)$ group when one encircles the singularities. In the second order canonical ADM formulation [3in appears which is described in Sec. 2. Such a problem occurs in determining the conformal factor $e^{2 \sigma}$ in the $\mathrm{ADM}$ metric

$$
d s^{2}=-N^{2} d t^{2}+e^{2 \sigma}\left(d z+N^{z} d t\right)\left(d \bar{z}+N^{\bar{z}} d t\right) .
$$

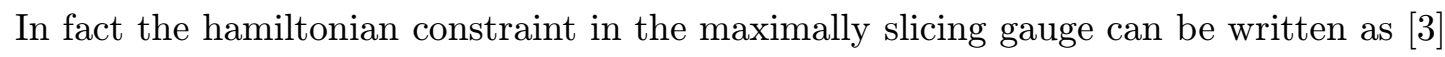

\footnotetext{
${ }^{*}$ Speaker.

${ }^{\dagger}$ Supported in part by MURST.
}

$$
4 \partial_{z} \partial_{\bar{z}} \phi=e^{\phi}+4 \pi \sum_{n} \delta^{2}\left(z-z_{n}\right)\left(-1+\mu_{n}\right)+4 \pi \sum_{B} \delta^{2}\left(z-z_{B}\right)
$$


where

$$
\phi=-2 \sigma+\ln \left[\frac{1}{2 \pi^{2}} \sum_{n} \frac{P_{n}}{\left(z-z_{n}\right)} \sum_{n} \frac{\bar{P}_{n}}{\left(\bar{z}-\bar{z}_{n}\right)}\right]
$$

being $z_{n}$ the particle positions, $P_{n}$ the canonically conjugate momenta and $4 \pi \mu_{n}$ the particle masses in Planck units. The $z_{B}$ are the zeros of $\sum_{n} P_{n} /\left(z-z_{n}\right)$ and are known as the apparent singularities. In eq.(12ij) one recognizes the Liouville equation with point sources.

The particle equations of motion in the relative coordinates can be written as [i.in

$$
\dot{z}_{n}^{\prime}=-\sum_{B} \frac{\partial \beta_{B}}{\partial \mu} \frac{\partial z_{B}^{\prime}}{\partial P_{n}^{\prime}}, \quad \dot{P}_{n}^{\prime}=\frac{\partial \beta_{n}}{\partial \mu}+\sum_{B} \frac{\partial \beta_{B}}{\partial \mu} \frac{\partial z_{B}^{\prime}}{\partial z_{n}^{\prime}}
$$

where $\beta_{B}$ are the accessory parameters related to the apparent singularities. One is faced with the problem of proving the hamiltonian nature of eqs.(i hamiltonian. As we shall see this problem has a straightforward solution if one assumes the validity of Polyakov's conjecture to which now we come.

2. Polyakov [i] put forward the following conjecture on the accessory parameters $\beta_{n}$ which appear in the solution of the $S U(1,1)$ Riemann-Hilbert problem

$$
-\frac{1}{2 \pi} d S_{P}=\sum_{n} \beta_{n} d z_{n}+c . c .
$$

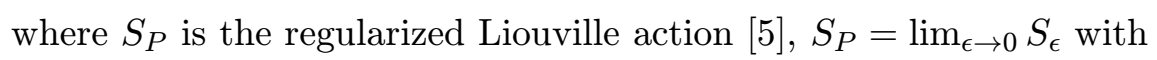

$$
\begin{gathered}
S_{\epsilon}[\phi]=\frac{i}{2} \int_{X_{\epsilon}}\left(\partial_{z} \phi \partial_{\bar{z}} \phi+\frac{e^{\phi}}{2}\right) d z \wedge d \bar{z}+\frac{i}{2} \sum_{n} g_{n} \oint_{\gamma_{n}} \phi\left(\frac{d \bar{z}}{\bar{z}-\bar{z}_{n}}-\frac{d z}{z-z_{n}}\right) \\
+\frac{i}{2} g_{\infty} \oint_{\gamma_{\infty}} \phi\left(\frac{d \bar{z}}{\bar{z}}-\frac{d z}{z}\right)-\pi \sum_{n} g_{n}^{2} \ln \epsilon^{2}-\pi g_{\infty}^{2} \ln \epsilon^{2}, \quad \text { where } \quad d z \wedge d \bar{z}=-2 i d x \wedge d y
\end{gathered}
$$

and $X_{\epsilon}$ is the disk of radius $1 / \epsilon$ in the complex plane from which disks of radius $\epsilon$ around all singularities have been removed; $\gamma_{n}$ are the boundaries of the small disks and $\gamma_{\infty}$ is the boundary of the large disk. In eq. (店) $S_{P}$ has to be computed on the solution of the inhomogeneous Liouville equation which arises from the minimization of the action i.e.

$$
4 \partial_{z} \partial_{\bar{z}} \phi=e^{\phi}+4 \pi \sum_{n} g_{n} \delta^{2}\left(z-z_{n}\right)
$$

with behavior at infinity $\phi=-g_{\infty} \ln z \bar{z}+O(1)$. Such a conjecture plays an important role

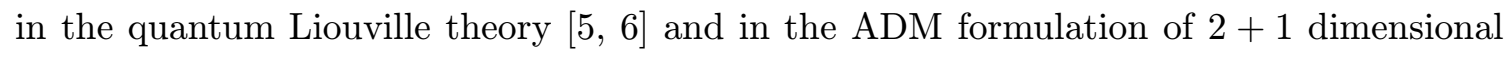

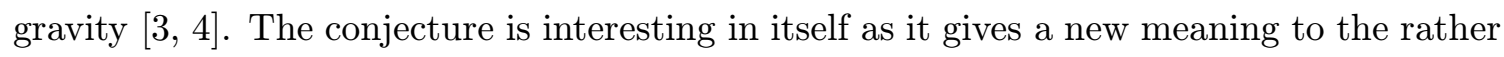
elusive accessory parameters [ئi $\left[\begin{array}{l}\overline{1} \\ 1\end{array}\right]$ of the Riemann-Hilbert problem. In particular it implies that the form $\omega=\sum_{n} \beta_{n} d z_{n}+$ c.c. is exact. Zograf and Takhtajan [i, provided a proof of eq. $\left(\underline{\overline{\underline{D}}}_{1}^{\prime}\right)$ for parabolic singularities. In addition they remark that the same technique can be applied when some of the singularities are elliptic of finite order. On the other hand in $2+1$ gravity one is faced with general elliptic singularities and here the mapping technique

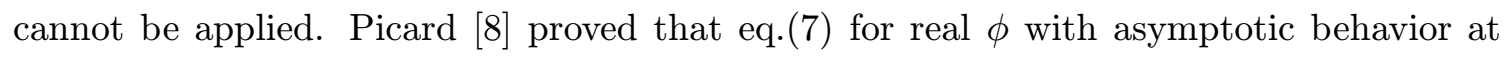


infinity $\phi(z)=-g_{\infty} \ln (z \bar{z})+O(1),-1<g_{n}, \quad 1<g_{\infty}$ and $\sum_{n} g_{n}+g_{\infty}<0$ admits one and only one solution (see also [9i]). The interest of such results is that they solve the following variant of the Riemann-Hilbert problem: at $z_{1}, \ldots z_{n}$ we are given not with the monodromies but with the class, characterized by $g_{j}$, of the elliptic monodromies with the further request that all such monodromies belong to the group $S U(1,1)$. The last requirement is imposed by the fact that the solution of eq. $\left(\overline{\mathbf{T}}_{1}\right)$ has to be single valued.

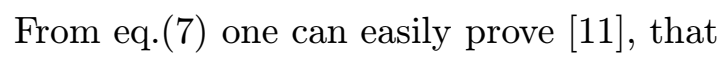

$$
e^{-\frac{\phi}{2}}=\frac{1}{\sqrt{8}\left|w_{12}\right|}\left[\bar{y}_{2}(\bar{z}) y_{2}(z)-\bar{y}_{1}(\bar{z}) y_{1}(z)\right]
$$

being $y_{1}, y_{2}$ two independent solutions of

$$
y^{\prime \prime}+Q(z) y=0 \quad \text { where } \quad Q(z)=\sum_{n}-\frac{g_{n}\left(g_{n}+2\right)}{4\left(z-z_{n}\right)^{2}}+\frac{\beta_{n}}{2\left(z-z_{n}\right)} .
$$

$w_{12}$ is the constant wronskian and the $\beta_{n}$ are the accessory parameters [i]

3. The result of Picard assures us that given the position of the singularities $z_{n}$ and the classes of monodromies characterized by the real numbers $g_{n}$ there exists a unique fuchsian equation which realizes $S U(1,1)$ monodromies of the prescribed classes. In particular the uniqueness of the solution of Picard's equation tells us that the accessory parameters $\beta_{i}$ are single valued functions of the parameter $z_{n}$ and $g_{n}$. We shall examine in this section how such dependence arises from the viewpoint of the imposition of the $S U(1,1)$ condition on the monodromies. Starting from the singularity in $z_{1}$ we can consider the canonical pair of solutions around $z_{1}, y_{1}^{1}=\zeta^{\frac{g_{m}}{2}+1} A(\zeta), y_{2}^{1}=\zeta^{-\frac{g_{m}}{2}} B(\zeta)$ with $A(\zeta)=1+O(\zeta), B(\zeta)=$ $1+O(\zeta), \zeta=z-z_{1}$, i.e. those solutions which behave as a single fractional power multiplied by an analytic function with first coefficient one. Let $\left(y_{1}, y_{2}\right)$ the solutions which realize $S U(1,1)$ around all singularities. Obviously all conjugations with any element of $S U(1,1)$ is still an equivalent solution in the sense that they provide the same conformal factor $\phi$. The canonical pairs around different singularities are linearly related i.e. $\left(y_{1}^{1}, y_{2}^{1}\right)=\left(y_{1}^{2}, y_{2}^{2}\right) C_{21}$. We fix the conjugation class by setting $\left(y_{1}, y_{2}\right)=\left(y_{1}^{1}, y_{2}^{1}\right) K$ with $K=\operatorname{diag}\left(k, k^{-1}\right)$ being the overall constant irrelevant in determining $\phi$. Moreover if the solution $\left(y_{1}, y_{2}\right)$ realizes $S U(1,1)$ monodromies around all singularities also $\left(y_{1}, y_{2}\right) \times \operatorname{diag}\left(e^{i \alpha}, e^{-i \alpha}\right)$ accomplishes the same purpose being $\operatorname{diag}\left(e^{i \alpha}, e^{-i \alpha}\right)$ an element of $S U(1,1)$. Thus the phase of the number $k$ is irrelevant and so we can consider it real and positive. This choice of the canonical pairs is always possible in our case. If $D_{n}$ denote the diagonal monodromy matrices around $z_{n}$, we have that the monodromy around $z_{1}$ is $D_{1}$ and the one around $z_{2}$ is $M_{2}=K^{-1} C_{12} D_{2} C_{21} K$, where with $C_{12}$ we have denoted the inverse of the $2 \times 2$ matrix $C_{21}$. In the case of three singularities (one of them at infinity) by using the freedom on $K$ we can reduce $M_{2}$ to the $S U(1,1)$ form. The possibility of such a choice is assured by Picard's result and in this simple case also by the explicit solution in terms of hypergeometric functions [2, 130 . We come now to a qualitative description of the case of four singularities. We recall that the accessory parameters $\beta_{n}$ are bound by two algebraic relations known as Fuchs relations [i, $]$. Thus after choosing $M_{1}$ of the form $M_{1}=D_{1} K$, in imposing 
the $S U(1,1)$ nature of the remaining monodromies we have at our disposal three real parameters i.e. $k, \operatorname{Re} \beta_{3}$ and $\operatorname{Im} \beta_{3}$. It is sufficient to impose the $S U(1,1)$ nature of $M_{2}$ and $M_{3}$ as the $S U(1,1)$ nature of $M_{\infty}$ is a consequence of them. As the matrices $M_{n}=K^{-1} C_{1 n} D_{n} C_{n 1} K$ satisfy identically $\operatorname{det} M_{n}=1$ and $\operatorname{Tr} M_{n}=2 \cos \pi g_{n}$ we need to impose generically on $M_{2}$ only two real conditions e.g. $\operatorname{Re} b_{2}=\operatorname{Re} c_{2}$ and $\operatorname{Im} b_{2}=-\operatorname{Im} c_{2}$. The same for $M_{3}$. Thus is appears that we need to satisfy four real relations when we can vary only three real parameters. The reason why we need only three and not four is that for any solution of the fuchsian problem the following relation among the monodromy matrices is identically satisfied $D_{1} K M_{2} M_{3} M_{\infty}=1$. The above reported discussion can be put on rigorous grounds [i] $10 \overline{1}\left[\begin{array}{l}i \\ ]\end{array}\right.$ for any number of singularities by exploiting the existence and uniqueness of the solution and using some basic results of the theory of analytic function of several complex variables, reaching the result that $\beta_{n}$ are analytic functions of $z_{m}, \bar{z}_{m}$ in the neighborhood of any point of the complex plane, except for a finite number of points.

4. Being defined through a limit procedure the action $S_{P}$ is somewhat uncomfortable to work with. It is however possible, introducing a background field to rewrite $S_{P}$ as a simple integral. In the global coordinate system $z$ on $C$ one writes $\phi=\phi_{M}+\phi_{0}+\phi_{B}$ where $\phi_{B}$ is a background conformal factor which is regular and behaves at infinity like $\phi_{B}=-2 \ln (z \bar{z})+c_{B}+O(1 /|z|)$ while $\phi_{0}$ is given by

$$
\phi_{0}=\sum_{n} g_{n} \ln \left|z-z_{n}\right|^{2}-\alpha \phi_{B}+c_{0} \quad \text { where } \quad \alpha=-\left(\sum_{n} g_{n}+g_{\infty}-2\right) / 2 .
$$

Then we have for $\phi_{M}$

$$
4 \partial_{z} \partial_{\bar{z}} \phi_{M}=e^{\phi_{0}+\phi_{B}+\phi_{M}}+(\alpha-1) 4 \partial_{z} \partial_{\bar{z}} \phi_{B} .
$$

$\phi_{M}$ is a continuous function on the Riemann sphere. The action which generates the above equation is

$$
S=\int\left[\partial_{z} \phi_{M} \partial_{\bar{z}} \phi_{M}+\frac{e^{\phi}}{2}+2(\alpha-1) \phi_{M} \partial_{z} \partial_{\bar{z}} \phi_{B}\right] \frac{i d z \wedge d \bar{z}}{2} .
$$

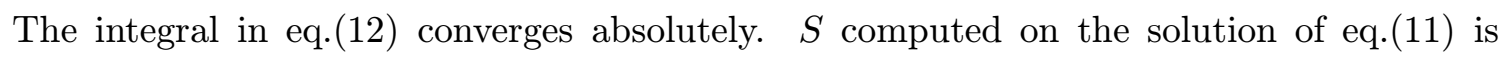

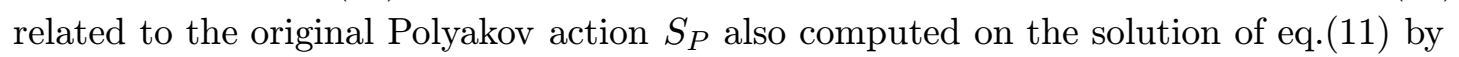

$$
\begin{aligned}
S_{P}=S & -(\alpha-1)^{2} \int \phi_{B} \partial_{z} \partial_{\bar{z}} \phi_{B} \frac{i d z \wedge d \bar{z}}{2}+2 \pi(\alpha-1)^{2} c_{B}+ \\
& +\pi \sum_{m} \sum_{n \neq m} g_{m} g_{n} \ln \left|z_{m}-z_{n}\right|^{2}+4 \pi c_{0}(1-\alpha) .
\end{aligned}
$$

Our aim now is to compute the derivative of $S_{P}$ with respect to $z_{n}$. Again computing the derivative of the new action $S$ is not completely trivial because one cannot take directly the derivative operation under the integral sign. In fact such unwarranted procedure would give rise to an integrand which is not absolutely summable. One can device however a

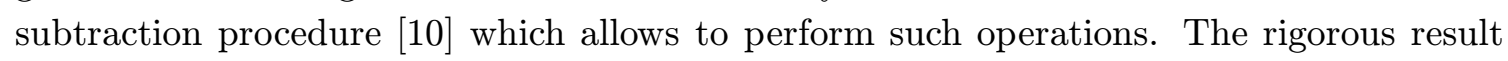

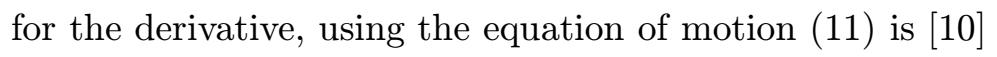

$$
\frac{\partial S}{\partial z_{m}}=\lim _{\epsilon \rightarrow 0} \int_{X_{\epsilon}}\left[\partial_{z}\left(\frac{\partial \phi_{M}}{\partial z_{m}} \partial_{\bar{z}} \phi_{M}\right)+\partial_{\bar{z}}\left(\frac{\partial \phi_{M}}{\partial z_{m}} \partial_{z} \phi_{M}\right)+\frac{\partial \phi_{0}}{\partial z_{m}} \frac{e^{\phi}}{2}\right] \frac{i d z \wedge d \bar{z}}{2}
$$


which can be computed by using eq.(定运) to obtain

$$
\frac{\partial S}{\partial z_{m}}=-i g_{m} \lim _{\epsilon \rightarrow 0} \oint_{\gamma_{\epsilon}} \frac{1}{z-z_{m}} \partial_{z}\left(\phi_{M}-(\alpha-1) \phi_{B}\right) d z .
$$

Using $\phi_{M}-(\alpha-1) \phi_{B}=\phi-\sum_{n} g_{n} \ln \left|z-z_{n}\right|^{2}$ and the expansion of $A=1+c_{1} \zeta+\cdots$ and $B=1+c_{2} \zeta+\cdots$ which are obtained by substituting into the differential equation ( $\left.\bar{q}_{1}^{\prime}\right)$ to obtain

$$
c_{1}=-\frac{\beta_{m}}{2\left(g_{m}+2\right)} \quad \text { and } \quad c_{2}=\frac{\beta_{m}}{2 g_{m}}
$$

finally we have

$$
\frac{\partial S}{\partial z_{m}}=-2 \pi \beta_{m}-2 \pi \sum_{n, n \neq m} \frac{g_{m} g_{n}}{z_{m}-z_{n}}
$$

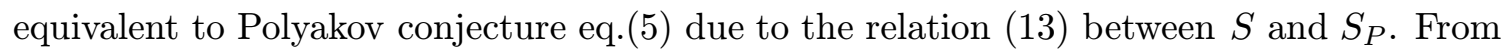
eqs. $\left(i_{0}^{1}, \underline{v}_{1}^{\prime}\right)$ we see that the hamiltonian is given by $H=\frac{1}{2 \pi} \frac{\partial S_{P}}{\partial \mu}$, because

$$
\frac{\partial H}{\partial P_{n}^{\prime}}=-\sum_{B} \frac{\partial \beta_{B}}{\partial \mu} \frac{\partial z_{B}^{\prime}}{\partial P_{n}^{\prime}} \quad \text { and } \quad-\frac{\partial H}{\partial z_{n}^{\prime}}=\frac{\partial \beta_{n}}{\partial \mu}+\sum_{B} \frac{\partial \beta_{B}}{\partial \mu} \frac{\partial z_{B}^{\prime}}{\partial z_{n}^{\prime}} .
$$

Acknowledgments We are grateful to Mauro Carfora for pointing out to us reference [9ī] and for useful discussions.

\section{References}

[1] A.M. Polyakov as reported in refs. [15i=1,

[2] A. Bellini, M. Ciafaloni, P. Valtancoli, Physics Lett. B 357 (1995) 532; Nucl. Phys. B 462 (1996) 453; M. Welling, Class. Quantum Grav. 13 (1996) 653; Nucl. Phys. B 515 (1998) 436.

[3] P. Menotti, D. Seminara, Ann. Phys. 279 (2000) 282; Nucl. Phys. (Proc. Suppl.) 88 (2000) 132.

[4] L. Cantini, P. Menotti, D. Seminara, Class. Quantum Grav. 18 (2001) 2253.

[5] L. A. Takhtajan, Mod. Phys. Lett. A11 (1996) 93; "Topics in quantum geometry of Riemann surfaces: two dimensional quantum gravity", Proc. Internat. School Phys. Enrico Fermi, 127, IOS, Amsterdam, 1996.

[6] P. G. Zograf, L. A. Takhtajan, Math. USSR Sbornik 60 (1988) 143; Math. USSR Sbornik 60 (1988) 297.

[7] M. Yoshida, "Fuchsian differential equations", Fried. Vieweg \& Sohn, Braunschweig (1987); K. Okamoto, J. Fac. Sci. Tokio Univ. 33 (1986) 575;J. A. Hempel, Bull. London Math. Soc. 20 (1988) 97.

[8] E. Picard, Compt. Rend. 116 (1893) 1015; J. Math. Pures Appl. 4 (1893) 273 and (1898) 313; Bull. Sci. math. XXIV 1 (1900) 196.

[9] M. Troyanov, Trans. Am. Math. Soc. 324 (1991) 793.

[10] L. Cantini, P. Menotti, D. Seminara, Phys. Lett. B 517 (2001) 203.

[11] H. Poincaré, J. Math. Pures Appl. (5) 4 (1898) 137. 